\title{
ESTIMULACIÓN TÉRMICA Y ANALGESIA HIPNÓTICA PARA LA REGULACIÓN AUTONÓMICA E INFLAMATORIA EN PACIENTES CON DOLOR CRÓNICO
}

\author{
Sandra Nayeli Vergara Aguirre, David Alberto Rodríguez Medina, Benjamín Domínguez Trejo, Li Erandi \\ Tepepa Flores, Astrid Vergara Erdosay, Noemí Guidone Mariotti y Jorge Rafael Hernández Santos \\ UNAM, ISSSTE, UNICATT \\ México
}

\section{RESUMEN}

El dolor crónico (DC) tiene implicaciones multidimensionales en la etiología, la evaluación y el tratamiento que hace necesario un abordaje interdisciplinario. Diversos estudios en dolor crónico sugieren una actividad de muy baja frecuencia en la variabilidad de la frecuencia cardiaca (VFC) asociada a la actividad simpática, y bajo nivel de actividad parasimpática (coherencia de alta VFC). El propósito de esta investigación es evaluar el efecto de la estimulación térmica en manos y el uso de la analgesia hipnótica en la actividad autonómica y la concentración de IL-6 salival en pacientes con DC no oncológico. Mediante un muestreo no probabilístico intencional, participaron nueve mujeres (edad $\bar{X}=58.22$ ) con diagnóstico de dolor crónico, quienes recibieron un tratamiento psicológico de sesión única de 15 minutos. Para el análisis de resultados se utilizó un diseño prospectivo pre-experimental para la muestra salival de IL-6 y temperatura periférica, y un diseño de medidas repetidas para los porcentajes de coherencia cardíaca (alta, baja y muy baja). Los resultados sugieren que la intervención psicológica no tuvo efecto estadísticamente significativo en la IL-6 ( $Z$ = $-0.508, p$ = 0.611). Sin embargo, se encontró un incremento de temperatura periférica en la mano dominante estadísticamente significativo pre-post intervención $(Z=-2.197, p=0.028$ ). Asimismo, la coherencia cardiaca alta incrementó con la intervención psicológica $(X 2$ $=6.643(2), p=0.036)$, en especial después del ejercicio de analgesia hipnótica $(Z=-2.197, p=0.028)$. La intervención psicológica en pacientes con $\mathrm{DC}$ produjo algunos cambios en la actividad autonómica. Se destaca su utilidad clínica como un procedimiento no invasivo para el manejo integral del paciente con dolor crónico en la evaluación y el tratamiento del DC.

Palabras Clave:

manejo del dolor, coherencia cardíaca, IL-6, temperatura periférica.

\section{THERMAL STIMULATION AND HYPNOTIC ANALGESIA FOR AUTONOMIC AND INFLAMMATORY REGULATION IN PATIENTS WITH CHRONIC PAIN}

\begin{abstract}
Chronic Pain (CP) has multidimensional implications in the etiology, evaluation and treatment that require an interdisciplinary approach. Several studies in chronic pain suggest a very low frequency activity in the variability of heart rate (HRV) associated with sympathetic activity, and low level of parasympathetic activity (consistency of high HRV). The purpose of this investigation was to evaluate the effect of the thermal stimulation in hands and the use of hypnotic analgesia on the autonomic activity and the concentration of salivary IL-6 in patients with non-oncological CP. Using an intentional non-probabilistic sampling, nine women participated (age $\bar{X}=58.22$ ) with a diagnosis of CP who received a psychological treatment of a single session of 15 minutes. For the analysis of results, a prospective, pre-experimental design was used for the salivary sample of IL- 6 and peripheral temperature; and a design of repeated measures for the percentages of Cardiac Coherence (high, low and very low). The results suggest that the psychological intervention had no statistically significant effect on IL-6 $(Z=-0.508, p=0.611)$. However, an increase in peripheral temperature was found in the statistically significant dominant hand pre-post intervention $(Z=-2,197, p=0.028)$. Likewise, the High Cardiac Coherence increased with the psychological intervention ( $X 2=6.643(2), p=0.036$ ), especially after the exercise of Hypnotic Analgesia $(Z=-2.197, p=0.028)$. Psychological intervention in patients with $C P$ produced some changes in autonomic activity. Its clinical usefulness is highlighted as a non-invasive procedure for the integral management of the patient with chronic pain in the evaluation and treatment of $C P$.
\end{abstract}

Keywords:

Non-invasive intervention of pain, cardiac coherence, IL-6, peripheral temperature.

Bitácora del Artículo:

| Recibido: 20 de Agosto de 2018 | Aceptado: 1 noviembre de 2018 | Publicado en línea: Julio-Diciembre de 2018 | 


\title{
Autoría y Derechos de Propiedad Intelectual
}

\section{ESTIMULACIÓN TÉRMICA Y ANALGESIA HIPNÓTICA PARA LA REGULACIÓN AUTONÓMICA E INFLAMATORIA EN PACIENTES CON DOLOR CRÓNICO}

\author{
Sandra Nayeli Vergara Aguirre, David Alberto Rodríguez Medina, Benjamín Domínguez Trejo, Li Erandi \\ Tepepa Flores, Astrid Vergara Erdosay, Noemí Guidone Mariotti y Jorge Rafael Hernández Santos \\ UNAM, ISSSTE, UNICATT \\ México
}

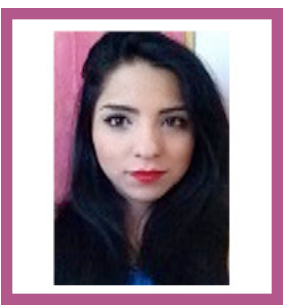

Sandra Nayeli Vergara Aguirre FES Zaragoza, UNAM

Correo: zandra_vergara@hotmail.com

Psicóloga egresada de la Facultad de Estudios Superiores Zaragoza.

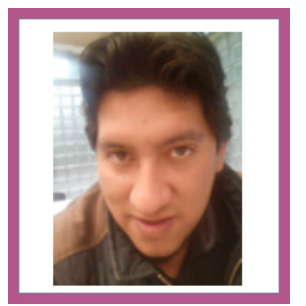

David Alberto Rodríguez Medina Facultad de Psicología, UNAM Correo: psic.d.rodriguez@comunidad. unam.mx

Candidato a Dr. en Psicología y Salud, Facultad de Psicología, UNAM. Reconocimiento de primer lugar en Enfermedades Crónicas No Transmisibles durante el II Foro Estatal de Investigación Clínica, 2016. Primer Lugar del $6^{\circ}$ Coloquio de Estudiantes en Neurociencias, categoría Doctorado, de la UAM, 2018.

Ver más... Ver más...

\section{CONTRIBUCIÓN DE LOS AUTORES}

Sandra Vergara participó en el desarrollo del proyecto, efectuó la instrumentación del equipo de retroalimentación, toma de muestras salivales y la aplicación del perfil psicofisiológico. Capturó y creó la base de datos | David Rodríguez participó en el desarrollo del artículo. Hizo el análisis de datos y redactó los resultados | Benjamín Domínguez Trejo coordinó el desarrollo del proyecto y el trabajo de campo I Li Tepepa participó en la instrumentación del equipo de retroalimentación y en la aplicación del perfil psicofisiológico | Astrid Erdosay identificó a las pacientes que cumplían con los criterios de inclusión y las contactó | Noemí Mariotti participó en el desarrollo documental de investigación y conducción de sesiones clínicas.

\section{AGRADECIMIENTOS}

Al maestro Gerardo Leija. Al biólogo Ricardo Márquez. A la bióloga Gabriela Hurtado Alvarado: Área de Neurociencias del Departamento de Biología de la Reproducción, UAM-I. Con el financiamiento de los proyectos académicos: Proyecto UNAM-DGAPA-PAPIIT IN304515: "Biomarcadores (autonómicos e inmunológicos) como indicadores del componente emocional en el dolor crónico". Proyecto PAPIME-DGAPA-UNAM PE300716: "Fronteras de la psicología: citocinas y emociones".

\section{Datos DE FiLIACión de LS Autores}

Facultad de Estudios Superiores Zaragoza UNAM | Facultad de Psicología UNAM

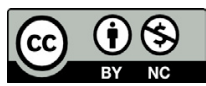

Copyright: () 2018 Vergara-Aguirre, S.N., Rodríguez-Medina, D.A., Domínguez-Trejo, B., Tepepa-Flores, L. E., Vergara-Erdosay, A. \& Guidone-Mariotti, N. Este es un artículo de acceso abierto distribuido bajo los términos de la licencia Creative Commons Reconocimiento-NoComercial 4.0 Internacional, por lo que su contenido gráfico y escrito se puede compartir, copiar y redistribuir total o parcialmente sin necesidad de permiso expreso de sus autoras con la única condición de que no se puede usar con fines directamente comerciales y los términos legales de cualquier trabajo derivado deben ser los mismos que se expresan en la presente declaración. La única condición es que se cite la fuente con referencia a la Revista Digital Internacional de Psicología y Ciencia Social y a sus autoras. 


\section{TABLA DE CONTENIDO}

\section{Diseño, 25}

Participantes, 25

Materiales e instrumentos, 25

Procedimiento, 25

Mediciones, 26

Análisis estadísticos, 26 


\section{INTRODUCCIÓN}

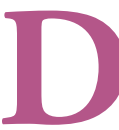

e acuerdo con la Asociación Internacional para el Estudio del Dolor (IASP, por sus siglas en inglés), el dolor es "una experiencia sensorial o emocional desagradable asociada a un daño real o potencial en un tejido, o descrito en términos de dicho daño" (IASP, 1994, p. 210), y es uno de los principales motivos de consulta en el primer nivel de atención médica (Dureja, Jain, Shetty, Mandal, Prabhoo \& Joshi et al. 2013). Este puede ser el resultado de una enfermedad subyacente, un problema de salud crónico o, en ocasiones, debido a razones desconocidas. Si el dolor persiste más allá del evento agudo que lo originó, del tiempo normal de curación, o se produce junto a una condición de salud crónica, pierde sus características adaptativas y se convierte en una enfermedad recalcitrante (Ruvalcaba \& Domínguez, 2009). El dolor crónico (DC) —a menudo descrito como un dolor de larga duración- tiene implicaciones multidimensionales en la etiología, la evaluación y el tratamiento (Ruvalcaba \& Domínguez, 2009; González, 2014).

EI DC ha sido considerado como un problema mundial de salud pública (Jensen, Gottrup, Kasch, Nikolajsen, Terkelsen \& Witting, 2001, como se citó en Covarrubias, Guevara, Gutiérrez, Betancourt \& Córdova, 2010; González, 2014). Se estima que dicho padecimiento afecta entre 25 a 29\% de la población mundial (Harstall \& Ospina, 2003), y en algunos reportes incluso se ha documentado una prevalencia que oscila entre 8 y $80 \%$ (Covarrubias, Guevara, Gutiérrez, Betancourt \& Córdova, 2010; González, 2014). Se calcula que en México más de 28 millones de habitantes padece este tipo de dolor; no obstante, se carece de estudios epidemiológicos que respalden dicho dato en la población mexicana (Covarrubias, Guevara, Gutiérrez, Betancourt \& Córdova, 2010). Un estudio epidemiológico nacional de 2007 (Barragán, Mejía \& Gutiérrez) sugiere que la prevalencia del DC en los adultos mayores es de $41.5 \%$; siendo las mujeres las más afectadas $(48.3 \%)$, por lo que dichos resultados proponen que la prevalencia del DC en México se incrementa en los grupos etarios de mayor edad.

Se ha documentado que el modelo biomédico es insuficiente para explicar el origen y tratamiento del DC ante las diferencias individuales en la práctica clínica (Cheatle, 2016; Bevers, Watts, Kishino \& Gatchel, 2016); por tanto, el enfoque biopsicosocial de dolor y la discapacidad, hoy es ampliamente aceptado para la comprensión y el abordaje del DC, porque reconoce la interacción compleja y dinámica entre factores neurofisiológicos (centrales y periféricos), psicológicos, ambientales y sociales como mecanismo subyacente que perpetúa e incluso empeora la intensidad del DC (Gatchel, McGeary, McGeary \& Lippe, 2014). Una actualización reciente del modelo biopsicosocial fue desarroIlada por Bevers, Watts, Kishino \& Gatchel (2016), en la que incorporan elementos de vulnerabilidad genética como la expresión de receptores a la citoquina inflamatoria interleucina 6 (IL-6), vulnerabilidad biológica (como trastornos endocrinológicos), trastornos psicológicos (influenciados por la actividad autonómica del distrés psicológico) y la vulnerabilidad social (por ejemplo, escaso apoyo social).

Diversos estudios apoyan la tesis de la participación de la IL-6 en la transición de la actividad inflamatoria aguda a la inflamación crónica para desarrollar DC (Fischer, Elfving, Lund \& Wegener, 2015; Karshikoff, Jensen, Kosek, Kalpouzos, Soop, Ingvar, et al., 2016; Lasselin, Lekander, Axelsson \& Karshikoff, 2018). Las citoquinas proinflamatorias como la IL-6 es mediada por la activación prolongada del eje hipofisiario-pituitario-adrenal, el cual induce una alta activación simpática, manteniendo al individuo hiperalerta, con fatiga crónica, problemas de sueño y estado anímico negativo, por lo que la evaluación de aspectos psicofisiológicos en el paciente con DC resulta indicada. La interacción entre la actividad elevada autonómica y la actividad inflamatoria permite un campo de competencia para el psicólogo especialista en manejo del dolor.

Por su parte, la experiencia dolorosa se asocia con el incremento de la actividad simpática y un decremento de la actividad parasimpática. Para regular la actividad autonómica asociada al dolor se han desarrollado, aplicado y adaptado diversas intervenciones psicofisiológicas como la expresión emocional escrita (Pennebaker, 1997), las técnicas de relajación (Skinner, Wilson \& Turk, 2012; Jensen \& Turk, 2014) o la analgesia hipnótica (AH) (Dillworth, Mendoza \& Jensen, 2011; Skinner, Wilson \& Turk, 2012; Jensen \& Turk, 2014).

La evidencia acumulada en el impacto neurofisiológico de la $\mathrm{AH}$ ha guiado el trabajo teórico y clínico para evaluar su efectividad en la reducción de la intensidad, duración y frecuencia del DC, así como para disminuir el uso de analgésicos en comparación con pacientes que no reciben tratamiento de este tipo, e incluso, tiene una eficacia similar a otros tratamientos que comparten mecanismos hipnóticos, como la relajación 
muscular progresiva, la retroalimentación biológica y el entrenamiento autógeno (Dillworth \& Jensen, 2010).

La $\mathrm{AH}$ promueve un efecto en la regulación de la variabilidad de la frecuencia cardíaca (VFC), una medida sensible al balance autonómico, al inducir un aumento del tono parasimpático y una disminución del tono simpático (Yüksel, Ozcan \& Dane, 2013); incluso diversos autores sugirieren que la reactividad de la rama parasimpática del sistema nervioso autónomo (SNA) reflejada en la VFC podría formar parte de una medida cuantitativa en tiempo real de la profundidad hipnótica (Diamond, Davis \& Howe, 2008; citado en Boselli et al., 2017). Además, una medida específica derivada de la VFC es la coherencia cardíaca (CC), mediante la cual estados emocionales específicos se reflejan en los patrones o la estructura de la forma de la onda de la VFC, independientemente de la cantidad de fluctuaciones en la frecuencia cardíaca que pueda presentar el individuo: "Un ritmo cardíaco coherente se define como una señal relativamente armónica (similar a una onda sinusoidal) con un pico muy estrecho de gran amplitud en la región de baja frecuencia (típicamente alrededor de $0.1 \mathrm{~Hz}$ ) del espectro de potencia sin picos importantes en las otras bandas" (McCraty \& Zayas, 2014, p. 5).

Cuando el paciente experimenta un estado de relajación su temperatura periférica y la CC de la VFC de alta frecuencia incrementan. Sin embargo, si el paciente no se beneficia con estos procedimientos se puede recurrir a procedimientos de estimulación térmica local cuyo mecanismo en la actividad simpática se ha documentado (Johnson \& Kellogg, 2010). Una estrategia para ello es la estimulación térmica corporal (Lee, Park \& Kim, 2011), la cual ha documentado efectos en la VFC de alta frecuencia, asociada a un predominio parasimpático.

Se han documentado las vías termosensoriales y circuitos neurales implicados en la disminución de estados afectivos negativos, promoviendo estados positivos y el involucramiento social, sin la mediación de la conciencia (Raison, Hale, Williams, Wager \& Lowry, 2015). Sin embargo, se desconoce si la estimulación térmica en pacientes con DC puede regular la actividad autonómica asociada al dolor, disminuyendo la actividad simpática (CC de muy baja frecuencia) e incrementando la respuesta parasimpática (CC de alta frecuencia y aumento de temperatura periférica).

La presente investigación tuvo como objetivo evaluar el efecto de la estimulación térmica en manos y el uso de la analgesia hipnótica con sugerencias no relacionadas con el dolor en pacientes con dolor crónico no oncológico en: 1) la actividad autonómica asociada a la actividad simpática (disminución de la CC de la VFC de muy baja frecuencia) y promover la actividad autonómica parasimpática (incremento de temperatura periférica y la CC de alta frecuencia de la VFC), y 2) disminuir la concentración de IL-6 salival.

\section{Método}

\section{Diseño}

Se hizo un estudio prospectivo, trasversal, pre-experimental en el periodo comprendido de mayo a diciembre de 2016.

\section{Participantes}

Se incluyeron nueve pacientes de la Clínica del Dolor del CMN "20 de Noviembre", del ISSSTE, con diagnóstico de dolor crónico no oncológico. La muestra incluyó pacientes sin patología oncológica, con edad que oscilaba entre los 30 y 70 a apoyo social percibido, índice de masa corporal normal y no fumadoras. Se excluyeron todas las pacientes que presentaran diabetes mellitus, asma, falla cardiaca congestiva, historia reciente de cáncer, artritis reumatoide, enfermedad cardiovascular, enfermedad obstructiva pulmonar, con marcapaso instalado, que estuviera ingiriendo medicamentos con propiedades inmunomoduladoras, con infección aguda (estomacal, respiratoria, etcétera), sordera o diagnóstico psiquiátrico. En la tabla 1 se indican las características sociodemográficas de la muestra.

\section{Tabla 1}

Características de la muestra.

\begin{tabular}{|c|c|c|}
\hline VARIABLES & & $M \quad$ (DE) \\
\hline \multirow[t]{2}{*}{$\begin{array}{l}\text { Edad } \\
\text { Sexo }\end{array}$} & & $\begin{array}{l}58.22(11.681) \\
-N(\%)\end{array}$ \\
\hline & Mujeres & 9 (100\%) \\
\hline $\begin{array}{l}\text { Tratamiento } \\
\text { farmacológico }\end{array}$ & $\begin{array}{l}\text { Tiopentadol } \\
\text { Imipramina } \\
\text { Oxicodona }\end{array}$ & $\begin{array}{l}4(44.4 \%) \\
3(33.3 \%) \\
2(22.2 \%)\end{array}$ \\
\hline Diagnóstico & $\begin{array}{l}\text { Canal lumbar estrecho } \\
\text { Neuralgia de Arnold } \\
\text { Fibromialgia } \\
\text { Hemicolectomía } \\
\text { Neuralgia del trigémino } \\
\text { Síndrome hombro doloroso }\end{array}$ & $\begin{array}{l}4(44.4 \%) \\
1(11.1 \%) \\
1(11.1 \%) \\
1(11.1 \%) \\
1(11.1 \%) \\
1(11.1 \%)\end{array}$ \\
\hline $\begin{array}{l}\text { Apoyo social } \\
\text { percibido }\end{array}$ & $\begin{array}{l}\text { Apoyo social } \\
\text { Sin apoyo }\end{array}$ & $\begin{array}{l}8(88.9 \%) \\
1(11.1 \%)\end{array}$ \\
\hline Depresión & $\begin{array}{l}\text { Leve } \\
\text { Moderada }\end{array}$ & $\begin{array}{l}6(66.7 \%) \\
3(33.3 \%)\end{array}$ \\
\hline
\end{tabular}




\section{Materiales e instrumentos}

En la tabla 2 se muestra la descripción de los aparatos, materiales y medio de recolección de datos utilizados en la presente investigación.

\section{Procedimiento}

El protocolo de investigación fue evaluado y aprobado por el Comité de Ética y de Investigación del Centro Médico Nacional "20 de Noviembre", ISSSTE. Se informó a las pacientes en qué consistía el estudio de manera individual, y si aceptaban participar firmaban un consentimiento informado y se presentaban a sus citas en el servicio de psicología de la clínica del dolor para recibir el tratamiento psicológico.

El protocolo de evaluación e intervención psicológica se estableció en el siguiente orden: 1) toma de muestra de saliva (preintervención); 2) medición de temperatura periférica en manos (preintervención); 3) aplicación de perfil psicofisiológico de respuesta al estrés (PPRE): la evaluación se hizo mediante un registro en tiempo real de la coherencia cardíaca, utilizando el equipo previamente mencionado, y por medio de la aplicación de un PPRE, con una duración de seis minutos repartidos en tres fases de dos minutos cada una: a) línea base, b) estímulo térmico y c) analgesia hipnótica; 4) medición de temperatura periférica (postintervención), y 5) toma de muestra de saliva (postintervención).

Primeramente debían cumplir con las recomendaciones del protocolo para hacer la toma de muestras de saliva (apéndice 1). Todas las evaluaciones se efectuaron entre las 7:00 y 9:00 a.m. La evaluación iniciaba con la recogida de muestra de saliva (figura 1A) y la medición de temperatura periférica en la mano dominante (figura 1B).

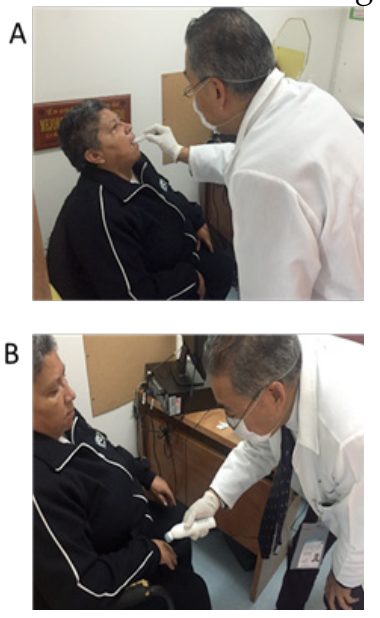

Figura 1

Recolecta de muestra salival (A) y registro de temperatura en mano dominante (B).
Tabla 2.

Descripción detallada de los aparatos, materiales y medio de recolección de datos.

\begin{tabular}{|c|c|}
\hline $\begin{array}{c}\text { CaRACTERÍSTICAS DE LA } \\
\text { MUESTRA. }\end{array}$ & Características \\
\hline $\begin{array}{l}\text { Equipo/Instrumento } \\
\text { emWave }{ }^{\circledR} \text { Desktop }\end{array}$ & $\begin{array}{l}\text { Software de retroalimentación } \\
\text { biológica en tiempo real, } \\
\text { entrenador de coherencia } \\
\text { cardiaca. Diseñado para } \\
\text { convertir la variabilidad de } \\
\text { la frecuencia cardiaca en } \\
\text { porcentajes de muy baja, baja } \\
\text { o alta coherencia (inducida } \\
\text { por la respiración voluntaria), } \\
\text { mediante modelos objetivos } \\
\text { medibles. }\end{array}$ \\
\hline Salivettes $®$ & $\begin{array}{l}\text { Tubo exterior grande con } \\
\text { un inserto pequeño y un } \\
\text { tapón de presión, utilizado } \\
\text { para almacenar muestras } \\
\text { de saliva recolectadas con } \\
\text { hisopos contenidos en los } \\
\text { Salivettes }{ }^{\circledR} \text { Se coloca un } \\
\text { hisopo de Salivettes }{ }^{\circledR} \text { en el } \\
\text { inserto pequeño y se congela } \\
\text { todo el tubo. Después } \\
\text { de la descongelación y la } \\
\text { centrifugación, la saliva se } \\
\text { conserva en el tubo exterior } \\
\text { grande. }\end{array}$ \\
\hline Termómetro digital HTD8808 & $\begin{array}{l}\text { Termómetro frontal infrarrojo } \\
\text { sin contacto. Permite medir a } \\
\text { una distancia de entre } 5 \mathrm{~cm} \text { a } \\
15 \mathrm{~cm} \text { en } \leq 1 \text { segundo. }\end{array}$ \\
\hline Compresa térmica & $\begin{array}{l}\text { Almohadilla térmica } \\
\text { instantánea, portátil y } \\
\text { reutilizable. Con un clic del } \\
\text { disco de metal que flota en } \\
\text { la solución salina, el Pad } \\
\text { llegará a } 54{ }^{\circ} \mathrm{C} \text {. La almohadilla } \\
\text { contiene una solución de } \\
\text { acetato de sodio en una bolsa } \\
\text { de plástico sellada. }\end{array}$ \\
\hline $\begin{array}{c}\text { Consentimiento informado y } \\
\text { entrevista estructurada }\end{array}$ & $\begin{array}{l}\text { Realizados ex profeso para } \\
\text { este estudio. }\end{array}$ \\
\hline
\end{tabular}

\begin{tabular}{|c|l|l|}
\hline Sensor & Respuesta medible & Ubicación corporal \\
\hline $\begin{array}{l}\text { Sensor de oreja } \\
\text { emWave }{ }^{8}\end{array}$ & $\begin{array}{l}\text { C o h e r e n c i a } \\
\text { cardíaca } \\
\text { (Alta; baja; muy } \\
\text { baja) }\end{array}$ & $\begin{array}{l}\text { Lóbulo de la oreja } \\
\text { izquierda }\end{array}$ \\
\hline
\end{tabular}


Apéndices

Apéndice 1.

Protocolo para la toma de muestras de saliva

Antes de efectuar la toma de mues-

b tra.

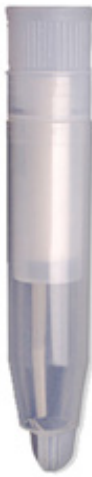

1.Evitar comer, beber, tomar chicles o limpiarse los dientes al menos 2 horas antes de la recogida de la muestra.

c 2.No recoger muestras cuando hay enfermedades bucales, inflamación o lesiones (contaminación por sangre).

3.Si existe contaminación visible por sangre esta muestra debe ser descartada.

4.Evitar el tabaco el día de la toma de muestra.

5.Evitar colocarse bálsamo para los labios resecos o lápiz labial.

Instrucciones para la toma de muestras.

1.Quite el tapón (a) y extraiga del Salivette $®$ el tubo interior (c).

2.Extraiga del interior del Salivette ${ }^{\circledR}$ la torunda (b).

3. Mantener la torunda en su boca hasta que note que no puede evitar tragar la saliva producida. Devolver la torunda (b) empapada en saliva al tubo interior, colocar éste en el portatubo (d) y cerrar bien el Salivette ${ }^{\circledR}$ con el tapón (a).

4. Rellenar una etiqueta del tubo (d) indicando el nombre del paciente.

5. Mantenga el Salivette ${ }^{\circledR}$ en refrigeración hasta devolverlo al Laboratorio.

Después se hizo la limpieza del lóbulo de la oreja izquierda y la colocación del sensor de emWave para hacer la medición de coherencia cardíaca; las indicaciones fueron: "coloque ambos pies sobre el piso, siéntese cómodamente, con la espalda recargada de modo adecuado en el respaldo de la silla, manténgase en silencio a menos que se le solicite lo contario, manténgase inmóvil y, en caso de sentir alguna molestia durante la evaluación, ésta puede terminar cuando lo desee". Al término de la evaluación de la línea base, se ndicó a la paciente que abriera lentamente los ojos hasta volver a situarse en el contexto del hospital y se le preguntaba cómo se sentía (figura 2).

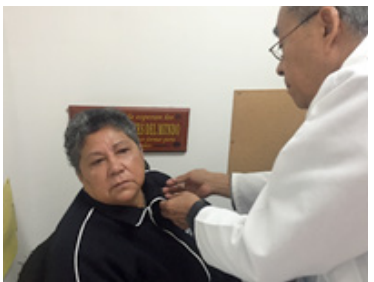

Colocación de sensor de variabilidad de la frecuencia cardiaca.

En seguida se procedió a indicarle que cerrara otra vez los ojos, se le colocaba en sus manos un cojín térmico a $54{ }^{\circ} \mathrm{C}$ que debía sostener, y a la par se le indicaba que evocara una situación agradable. Pasado el tiempo, se retiró el cojín térmico y se le preguntó acerca de las sensaciones experimentadas (figura 3).

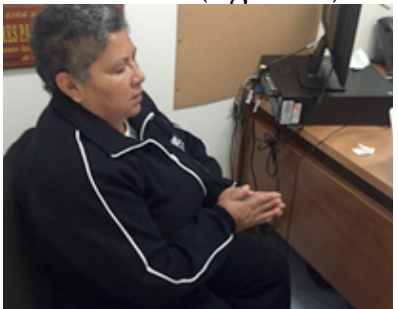

Figura 3.

Administración de la estimulación térmica en manos.

Luego se efectuó un procedimiento de analgesia hipnótica sin hacer referencia al dolor, en donde se invitaba a la paciente a enfocar su atención en la voz del instructor, incluyendo sugerencias para relajar los músculos, evocar el recuerdo de situaciones placenteras, hacer la difusión del calor experimentado recientemente y permitiéndole que recorriera lentamente todo su cuerpo, modulando la intensidad del calor/bienestar mediante metáforas (por ejemplo, con un control remoto), y creando sentimientos de autoeficacia con la proposición de que tal beneficio lo puede producir fácil y naturalmente cuando lo necesite. Por último, recibieron sugerencias posthipnóticas de seguir practicándolo en casa (figura 4).

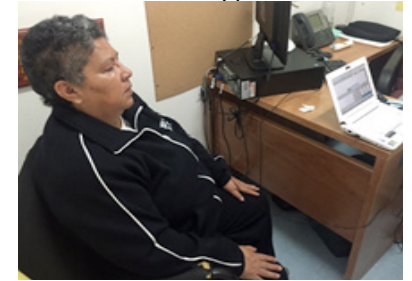

Figura 4.

Analgesia hipnótica.

Para finalizar, se retiraba el sensor, se hacía la medición de temperatura periférica (postintervención) y una toma de muestra de saliva (postintervención), se resolvían sus dudas y se le agradecía su colaboración (figura 5).

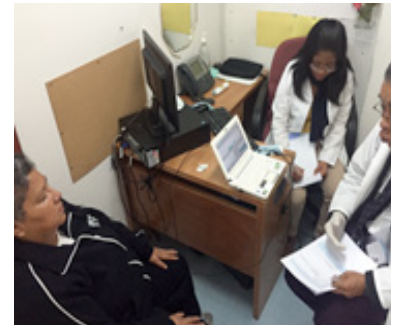

Figura 2 


\section{Figura 5}

Cierre de sesión.

Inmediatamente se colocaban las muestras de saliva en el refrigerador, para después llevarlas al laboratorio para su análisis (apéndice 2).

\section{Apéndice 2.}

Análisis de IL-6

La toma de muestra de saliva debió de estar perfectamente protocolizada y estandarizada para asegurar que las condiciones de recogida de la muestra fueron óptimas para su análisis.

Luego se centrifugaron durante 10 minutos a 3000 rpm. Se extrajeron los hisopos de los salivettes, el contenedor interno del tubo y se cerraron de nuevo; se recogió el suero y fue almacenado en un congelador Reveo a $-70{ }^{\circ} \mathrm{C}$, en tubos de plástico libres de pirógenos hasta su análisis en el laboratorio del Área de Neurociencias del Departamento de Biología de la Reproducción de la UAM-Iztapalapa.

Se determinó la concentración de IL-6 con un KIT Enzyme-Linked Immunosorbant Assay ( ELISA, por sus siglas en inglés) con las instrucciones del fabricante (Salimetrics, State College, PA) y se analizó con el siguiente procedimiento.

1. El antígeno (generador de anticuerpos, principalmente proteínas y polisacáridos que estimulan la producción de anticuerpos) se encuentra en microplaca.

2. El anticuerpo (respuestas del organismo ante la presencia de un agente infeccioso, los anticuerpos son moléculas proteicas secretadas por los plasmocitos; los anticuerpos utilizados en el método ELISA son de origen monoclonal o polidonal) en microplaca.

Se efectuó una dilución de 1:5 para las mediciones de muestras salivales de IL-6. El rango de detección para IL-6 fue de 0.2 a 3800 pg/ml. Las concentraciones de IL-6 se determinaron utilizando un contador de luminiscencia a $450 \mathrm{~nm}$ con un filtro secundario de 620 a $630 \mathrm{~nm}$.

Después se hizo la curva de ajuste estándar para obtener las concentraciones de IL- 6 de cada uno de los participantes en el estudio: 1) la adsorción de proteínas y el bloqueo de los sitios no ocupados con proteína neutral: se añadió muestra de antígeno para ser detectado en cada pocito; se incubó durante 30 minutos a $370{ }^{\circ} \mathrm{C}$; 2) se añadió solución de anticuerpo en cada pocito: se agregó un segundo anticuerpo primario contra el antígeno y conjugado con HRP anticuerpo secundario (mezcla de anticuerpos) en cada pocito; se incubó 30 minutos a $370{ }^{\circ} \mathrm{C}$; 3) se añadió enzima anticuerpo secundario conjugado en cada pocito y la reacción colorimétrica con sustrato apropiado: desarrollar reacción colorimétrica con sustrato apropiado; se incubó 15 minutos a temperatura ambiente, y 4) se leyó la absorbancia en espectrofotómetro ELISA: la reacción con $\mathrm{H} 2 \mathrm{~S} 043 \mathrm{M}$ y se cuantificaron los niveles de antígeno relativos.

\section{Mediciones}

Se utilizó un diseño prospectivo, pre-experimental para la muestra salival de IL-6 y temperatura periférica, y un Tabla 3

Definición conceptual y operacional de las variables utilizadas.

\begin{tabular}{|c|c|c|}
\hline \multicolumn{2}{|l|}{ VARIABLE } & $\begin{array}{l}\text { INSTRUMENTO DE } \\
\text { MEDICIÓN }\end{array}$ \\
\hline $\begin{array}{l}\text { Coherencia } \\
\text { cardíaca }\end{array}$ & $\begin{array}{l}\text { Estado de la variabilidad } \\
\text { de la frecuencia cardiaca } \\
\text { (VFC) inducido por la } \\
\text { respiración voluntaria, } \\
\text { que ocasiona un } \\
\text { aumento de la amplitud } \\
\text { del ritmo cardíaco, } \\
\text { una sincronización con } \\
\text { la respiración y por } \\
\text { tanto un estado de } \\
\text { bienestar psicológico. } \\
\text { La coherencia alta de } \\
\text { VFC está asociada con la } \\
\text { actividad parasimpática. }\end{array}$ & $\begin{array}{l}\mathrm{S} \text { o } \mathrm{f} \mathrm{t} \text { a } \mathrm{r} \text { e } \\
\text { Entrenador } \mathrm{de} \\
\text { coherencia del } \\
\text { ritmo cardíaco: } \\
\text { e m W a } \mathrm{m} \text { e } \\
\text { Desktop }\end{array}$ \\
\hline $\begin{array}{l}\text { Temperatura } \\
\text { periférica }\end{array}$ & $\begin{array}{l}\text { Medida autonómica } \\
\text { distal respecto } \\
\text { al corazón, cuyo } \\
\text { c o m p o r t a m i e } \mathrm{n} \text { t o } \\
\text { de vasoconstricción } \\
\text { (descenso de } \\
\text { t e m p e r a t u r a ) } \\
\text { está asociado al } \\
\text { aumento de actividad } \\
\text { simpática, mientras } \\
\text { que la vasodilatación } \\
\text { promueve el aumento } \\
\text { de la temperatura } \\
\text { inhibiendo la actividad } \\
\text { simpática facilitando la } \\
\text { actividad parasimpática. }\end{array}$ & $\begin{array}{l}\text { T e r m ó m e t r o } \\
\text { digital HTD8808 }\end{array}$ \\
\hline Interleucina 6 & $\begin{array}{l}\text { Mediador soluble de la } \\
\text { respuesta inmunológica } \\
\text { que incide en la } \\
\text { regulación de procesos } \\
\text { en nuestro organismo } \\
\text { ante la presencia de } \\
\text { estímulos estresantes } \\
\text { tanto físicos como } \\
\text { psicológicos. }\end{array}$ & $\begin{array}{c}\text { Véase el Apéndice } \\
2 .\end{array}$ \\
\hline
\end{tabular}

diseño de medidas repetidas para la VFC. En la tabla 3 se describen las variables de estudio y el instrumento de medición.

\section{Análisis estadísticos}


Para evaluar la significancia de los datos se utilizó la prueba estadística no paramétrica de Wilcoxon, que permitió evaluar las mediciones pre-post de IL-6 y temperatura periférica, mientras que para las mediciones de VFC se utilizó una prueba de Friedman para cada una de las coherencias de la VFC (alta, baja y muy baja). Se estableció un $\bar{X}=0.05$.

\section{Resultados}

La intervención psicológica no tuvo efecto estadísticamente significativo en la IL-6, Mdpre $=6.01 \mathrm{pg} / \mathrm{ml}$, Mdpost $=7.59 \mathrm{pg} / \mathrm{ml}(\mathrm{Z}=-0.508, \mathrm{p}=0.611)$.

Sin embargo, en la mano dominante se encontró un incremento de temperatura periférica estadísticamente significativo pre-post intervención de $\mathrm{Md}$ pre $=33.5{ }^{\circ} \mathrm{C}$ a $\mathrm{Md}$ post $=35.2{ }^{\circ} \mathrm{C}(\mathrm{Z}=-2.197, \mathrm{p}=0.028$; con un tamaño del efecto $r=-0.587$ ) (figura 6A).

A

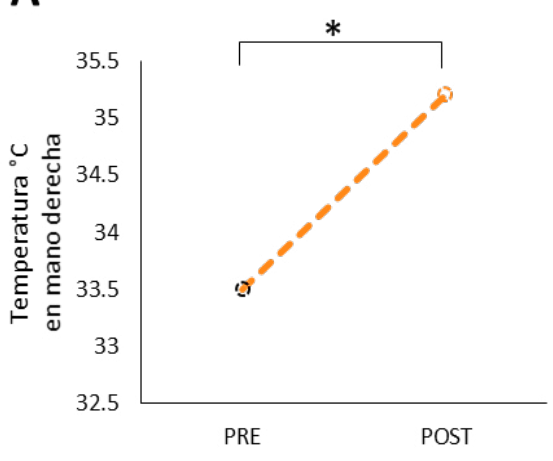

B

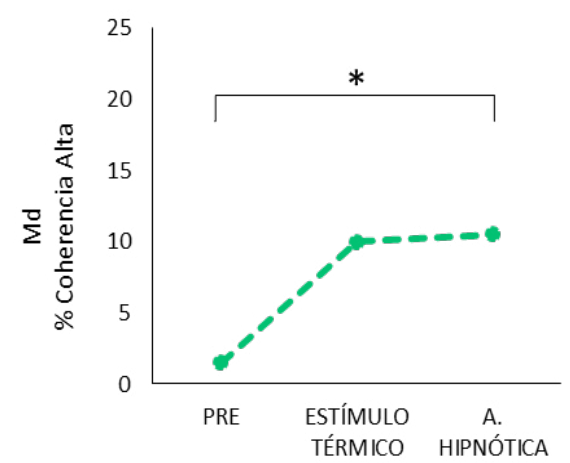

En las coherencias de la VFC incrementó la frecuencia alta con la intervención psicológica $(X 2=6.643$ $(2), p=0.036)$, de manera particular con el ejercicio de analgesia hipnótica $(Z=-2.197, p=0.028$; con un tamaño del efecto $r=-0.550$ ) (figura 6B), mientras que la coherencia baja no mostró cambios estadísticamente significativos (X2 = $2(2), p=0.368)$ (figura 6C), ni la coherencia muy baja (X2 $=0.581, p=0.748$ ) (figura 6D). En la figura 6 se ilustra la actividad autonómica subyacente al efecto de la intervención psicológica.

\section{Discusión}

Respecto a los objetivos planteados en este estudio clínico de explorar los efectos de la estimulación térmica (directa y evocada) y la analgesia hipnótica sin sugerencias alusivas al dolor, en la actividad autonómica y la concentración de IL-6 salival, se encontró que la intervención psicológica fue estadísticamente significativa en

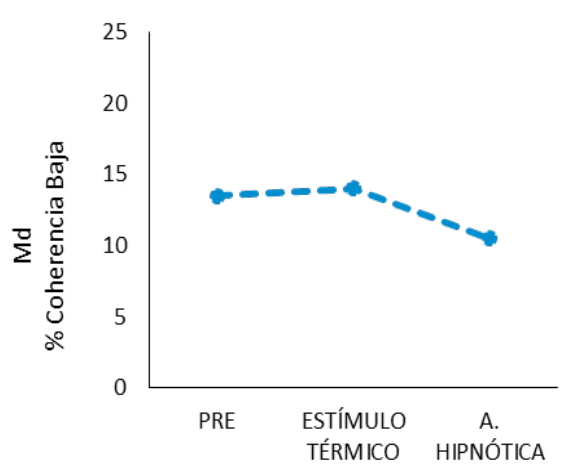

D

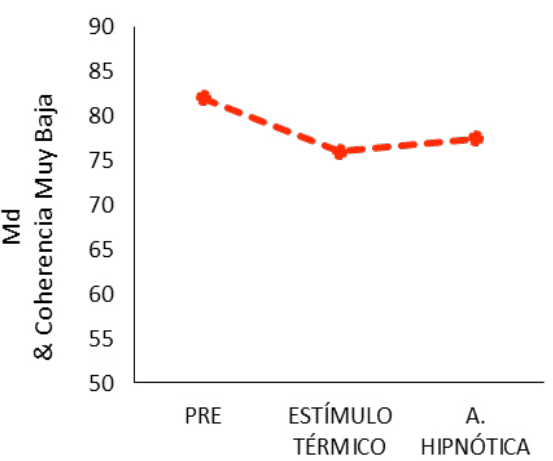

La intervención psicológica indujo algunos cambios autonómicos asociados estadísticamente significativos: (A) La temperatura periférica en la mano dominante se incrementó, (B) la coherencia de alta frecuencia de la variabilidad de la frecuencia cardíaca también aumentó (ambos indicadores de actividad parasimpática). Sin embargo, ni (C) la coherencia baja de la variabilidad de la frecuencia cardiaca, ni la coherencia muy baja (D) mostraron cambios estadísticamente significativos. ${ }^{*} \leq 0.05$.

Figura 6

Efectos de la intervención psicológica sobre la actividad autonómica. 
algunos indicadores de actividad parasimpática, como el incremento en la temperatura periférica y la coherencia de frecuencia alta de la VFC. Sin embargo, no fue posible modificar el nivel de IL-6 salival, y el nivel de la coherencia cardíaca de muy baja frecuencia siguió predominando. Esto significa que la intervención promovió el tono parasimpático, pero falta disminuir el tono simpático.

Friedman y Taub (1984, como se citó en Dillworth \& Jensen, 2010), en un estudio de $\mathrm{AH}$ con sugerencias relacionadas ya sea con o sin dolor, demostraron que no había diferencias estadísticamente significativas entre cuatro condiciones: inducción hipnótica estándar, inducción hipnótica estándar con imágenes térmicas (se pidió a las pacientes que imaginaran colocar sus manos en agua tibia y experimentaran el calor en la mano durante la hipnosis), retroalimentación biológica y relajación, porque todos los grupos de tratamiento fueron más efectivos que la condición de control de lista de espera. Con ello sugerían que dichas estrategias tienen efectos similares entre sí para la retroalimentación biológica o el entrenamiento en relajación. Sin embargo, debido a la falta de estudios que también hayan comparado estos tratamientos, cualquier conclusión extraída debe considerarse preliminar.

No obstante, con base en los hallazgos de la presente investigación clínica, se observó que en la condición de $\mathrm{AH}$ sin sugerencias alusivas al dolor, en particular se presentó un incremento en el porcentaje de CC, por lo que se considera que la hipnosis puede tener alguna ventaja sobre otras estrategias activas utilizadas. Asimismo, la hipótesis de que las emociones positivas autoinducidas aumentan la coherencia cardíaca en los procesos fisiológicos y que se refleja en el patrón del ritmo cardíaco (McCraty, Atkinson, Tomasino \& Bradley 2009; McCraty \& Childre 2010; McCraty \& Zayas, 2014;) es compatible con esta investigación, porque al inducir el estado hipnótico sin sugerencias de dolor se presentó un aumento de la CC de alta frecuencia de la VFC, en contraste a las anteriores condiciones en donde no se dirigía verbalmente a las pacientes.

Varios estudios psicológicos han reportado que el nivel basal alto de concentración de IL-6 influye de modo negativo en el efecto de los tratamientos psicológicos (Lasselin, Kemani, Kanstrup, Olsson, Axelsson, Andreasson, et al., 2016; Rodríguez-Medina, 2018; Rodríguez-Medina, Domínguez, Cortés, Cruz, Morales \& Leija, 2018). Para futuras investigaciones se sugiere considerar el nivel de IL-6 previo de cada participante, pues sólo dos pacientes lograron decrementar su actividad inflamatoria. Se sugiere también establecer criterios de inclusión basados en la evaluación previa en la capacidad del paciente para sostener la atención focalizada hacia la analgesia hipnótica (susceptibilidad hipnótica), e incluso evaluar la hipersensibilidad al estímulo térmico, el cual alcanza una temperatura máxima sin poder regularla con este material. Se ha documentado que pacientes con experiencias traumáticas tienen un nivel de concentración de IL-6 elevado, manteniendo una hiperactivación simpática con hiperestesia y dificultades de concentración para relajarse, entre otros síntomas de estrés postraumático. La valoración previa podría establecer prioridades de atención clínica tanto médica como psicológica.

Por último, dentro de las limitaciones del artículo se destaca que no se tuvo un tamaño de muestra representativo de la población, aleatorizado ni con etiología homogénea del diagnóstico, ni con grupo control, por lo que los resultados aquí mostrados sólo responden al comportamiento de dicha muestra y a pacientes con perfiles equivalentes. Se sugiere incorporar sesiones de entrenamiento en respiración profunda previas al uso de la $\mathrm{AH}$ para inducir aumentos relativos en la actividad parasimpática que están asociados con una mayor CC (McCraty, Atkinson, Tomasino \& Bradley 2009); así como calendarizar un periodo de seguimiento que permita evaluar los efectos psicológicos (conductas de ingesta de medicamento, interferencia con actividades cotidianas, estados afectivos negativos) y psicofisiológicos de manera longitudinal. Además, es esencial una mayor comprensión de las estrategias más efectivas de intervenciones psicológicas dirigidas a subgrupos específicos.

\section{Conclusiones}

La introducción del modelo biopsicosocial del DC en los últimos años estimuló el desarrollo de programas interdisciplinarios para el manejo terapéutico efectivo y rentable (Gatchel, McGeary, McGeary \& Lippe, 2014). El abordaje psicológico ha desarrollado estrategias alternativas eficaces para reducir la discapacidad y los aspectos psicosociales comórbidos en pacientes con dicha condición (Majeed, Ali \& Sudak, 2018), pero es porque las intervenciones psicológicas para el manejo del DC han mostrado en promedio un tamaño del efecto de pequeño a moderado (Ehde, Dillworth \& Turner, 2014), que surge la necesidad de adaptar nuevas estrategias para el abordaje integral del DC.

Por tanto, el uso de biomarcadores (autonómicos e inmunológicos) ofrece una opción objetiva, poco invasiva, con un elevado grado de exactitud y con alto valor pronóstico que ayuda a los profesionales de la salud a complementar los elementos para ofrecer intervenciones más efectivas para la modulación del dolor. 
Este estudio muestra que la $\mathrm{AH}$ sin sugerencias relacionadas con el dolor induce un aumento en la coherencia cardíaca de frecuencia alta y en la temperatura periférica, que se asocia a un dominio del sistema nervioso parasimpático, y que a su vez impacta de modo positivo en la modulación del dolor, el funcionamiento físico y emocional: también pueden utilizarse como una opción o como complemento del manejo farmacológico en pacientes con DC no oncológico.

Asimismo, estos resultados sugieren que la monitorización de la coherencia cardíaca proporciona una herramienta clínica objetiva para la medición de los cambios del proceso hipnótico. Sin embargo, es necesario hacer observaciones longitudinales del entrenamiento de la $\mathrm{AH}$ sosteniendo el estímulo térmico para evaluar el efecto en el predominio de la actividad autonómica prolongado en estas pacientes que influya de manera directa en la percepción del dolor y su correlato psicofisiológico.

\section{Referencias}

Barragán, A., Mejía, S. \& Gutiérrez, L. (2007). Dolor en adultos mayores de 50 años: prevalencia y factores asociados. Salud Pública de México, 49, 488-494.

Bevers, K., Watts, L., Kishino, N., \& Gatchel, R. (2016). The biopsychosocial model of the assessment, prevention, and treatment of chronic pain. US Neurology, 12(2), 98. doi: 10.17925/usn.2016.12.02.98.

Boselli, E., Musellec, H., Martin, L., Bernard, F., Fusco, N., \& Guillou, N. et al. (2017). Effects of hypnosis on the relative parasympathetic tone assessed by ANI (Analgesia/ Nociception Index) in healthy volunteers: a prospective observational study. Journal of Clinical Monitoring and Computing, 32(3), 487-492. doi: 10.1007/s10877-017-0056$\underline{5}$.

Cheatle, M. (2016). Biopsychosocial approach to assessing and managing patients with chronic pain. Medical Clinics of North America, 100(1), 43-53. doi: 10.1016/j. mcna.2015.08.007.

Covarrubias, A., Guevara, U., Gutiérrez, C., Betancourt, J. \& Córdova, J. (2010). Epidemiología del dolor crónico en México. Revista Mexicana de Anestesiología, 33(4), 207-213.

Dillworth, T., \& Jensen, M. P. (2010). The role of suggestions in hypnosis for chronic pain: a review of the literature. The Open Pain Journal, 3(1), 39-51.

Dillworth, T., Mendoza, M., \&Jensen, M. (2011). Neurophysiology of pain and hypnosis for chronic pain. Translational Behavioral Medicine, 2(1), 65-72. doi: 10.1007/s13142-0110084-5.

Dureja, G., Jain, P., Shetty, N., Mandal, S., Prabhoo, R., Joshi, M. \& Goswami, S., et al. (2013). Prevalence of chronic pain, impact on daily life, and treatment practices in India. Pain Practice, 14(2), 51-62. doi: 10.1111/papr.12132.

Ehde, D., Dillworth, T., \& Turner, J. (2014). Cognitive-behavioral therapy for individuals with chronic pain: Efficacy, innovations, and directions for research. American Psychologist, 69(2), 153-166. doi: 10.1037/a0035747.

Fischer, C., Elfving, B., Lund, S., \& Wegener, G. (2015). Behavioral and systemic consequences of long-term inflammatory challenge. Journal of Neuroimmunology, 288, 40-46. doi: 10.1016/j.jneuroim.2015.08.011.

Gatchel, R., McGeary, D., McGeary, C., \& Lippe, B. (2014). Interdisciplinary chronic pain management: Past, present, and future. American Psychologist, 69(2), 119-130. doi: 10.1037/a0035514.

González, M. (2014). Dolor crónico y psicología: actualización. Revista Médica Clínica Las Condes, 25(4), 610-617. doi: 10.1016/s0716-8640(14)70081-1.

Harstall, C., \& Ospina, M. (2003). How prevalent is chronic pain? Pain Clinical Update, 11(2), 1-4.

International Association for the Study of Pain [IASP] (1994). Part III. Pain Terms: A Current List with Definitions and Notes on Usage. En H. Merskey and N. Bogduk (eds.), Classification of chronic pain (209-214). Seattle, USA: IASP Press.

Jensen, M., \& Turk, D. (2014). Contributions of psychology to the understanding and treatment of people with chronic pain: Why it matters to ALL psychologists. American Psychologist, 69(2), 105-118. doi: 10.1037/a0035641.

Johnson, J., \& Kellogg, D. (2010). Local thermal control of the human cutaneous circulation. Journal of Applied Physiology, 109, 1229-1238. doi :10.1152/japplphysiol.00407.2010.

Karshikoff, B., Jensen, K., Kosek, E., Kalpouzos, G., Soop, A., \& Ingvar, M. et al. (2016). Why sickness hurts: A central mechanism for pain induced by peripheral inflammation. Brain, Behavior, and Immunity, 57, 38-46. doi: 10.1016/j. bbi.2016.04.001.

Lasselin, J., Kemani, M., Kanstrup, M., Olsson, G., Axelsson, J., Andreasson, A., \& Lekander, M., et al. (2016). Low-grade inflammation may moderate the effect of behavioral treatment for chronic pain in adults. Journal of Behavioral Medicine, 39(5), 916-924. doi: 10.1007/s10865-016-9769-z.

Lasselin, J., Lekander, M., Axelsson, J., \& Karshikoff, B. (2018). Sex differences in how inflammation affects behavior: What we can learn from experimental inflammatory models in humans. Frontiers in Neuroendocrinology, 50, 91-106. doi: 10.1016/j.yfrne.2018.06.005.

Lee, Y., Park, B., \& Kim, S. (2011). The effects of heat and massage application on autonomic nervous system. Yonsei Medical Journal, 52(6), 982-989. doi: 10.3349/ymj.2011.52.6.982.

Majeed, M., Ali, A., \& Sudak, D. (2018). Psychotherapeutic interventions for chronic pain: Evidence, rationale, and advantages. The International Journal of Psychiatry in Medicine. doi: 10.1177/0091217418791447.

McCraty, R., \& Childre, D. (2010). Coherence: bridging personal, social and global health. Alternative Therapies, 16(4), 10-24.

McCraty, R., \& Zayas, M. (2014). Cardiac coherence, selfregulation, autonomic stability, and psychosocial wellbeing. Frontiers in Psychology, 5, 1-13. doi: 10.3389/ fpsyg.2014.01090.

McCraty, R., Atkinson, M., Tomasino, D., \& Bradley, R. (2009). The coherent heart: heart-brain interactions, psychophysiological coherence, and the emergence of system-wide order. Integral Review, 5(2), 10-115.

Pennebaker, J. (1997). Opening Up: The Healing Power of Expressing 


\section{Emotions.}

Raison, C., Hale, M., Williams, L., Wager, T., \& Lowry, C. (2015). Somatic influences on subjective well-being and affective disorders: the convergence of thermosensory and central serotonergic systems. Frontiers in Psychology, 5, 1-22. doi: 10.3389/fpsyg.2014.01580.

Rodríguez-Medina, D. (2018). Dolor y termografía. Revista Digital Internacional de Psicología y Ciencia Social, 1(4), 109-117. doi: 10.22402/j.rdipycs.unam.4.1.2018.164.109-117.

Rodríguez-Medina, D., Domínguez, B., Cortés, P., Cruz, I., Morales, L. \& Leija, G. (2018). Biopsychosocial assessment of pain with thermal imaging of emotional facial expression in breast cancer survivors. Medicines, 5(2), 30. doi: 10.3390/ medicines5020030.
Ruvalcaba, G. y Domínguez, B. (2009). La terapia psicológica del dolor crónico. Psicología y Salud, 19 (2), 247-252.

Skinner, M., Wilson, H., \& Turk, D. (2012). Cognitive-behavioral perspective and cognitive-behavioral therapy for people with chronic pain: Distinctions, outcomes, and innovations. Journal of Cognitive Psychotherapy, 26, 93-113. doi:10.1891/0889-8391.26.2.93.

USA: The Guilford Press.

Yüksel, R., Ozcan, O., \& Dane, S. (2013). The effects of hypnosis on heart rate variability. International Journal of Clinical and Experimental Hypnosis, 61(2), 162-171. doi: 10.1080/00207144.2013.753826. 


\section{Meta-Análisis del Artículo}




\section{Dimensión Cuantitativa}

\section{Perfil de Evaluación entre pares}
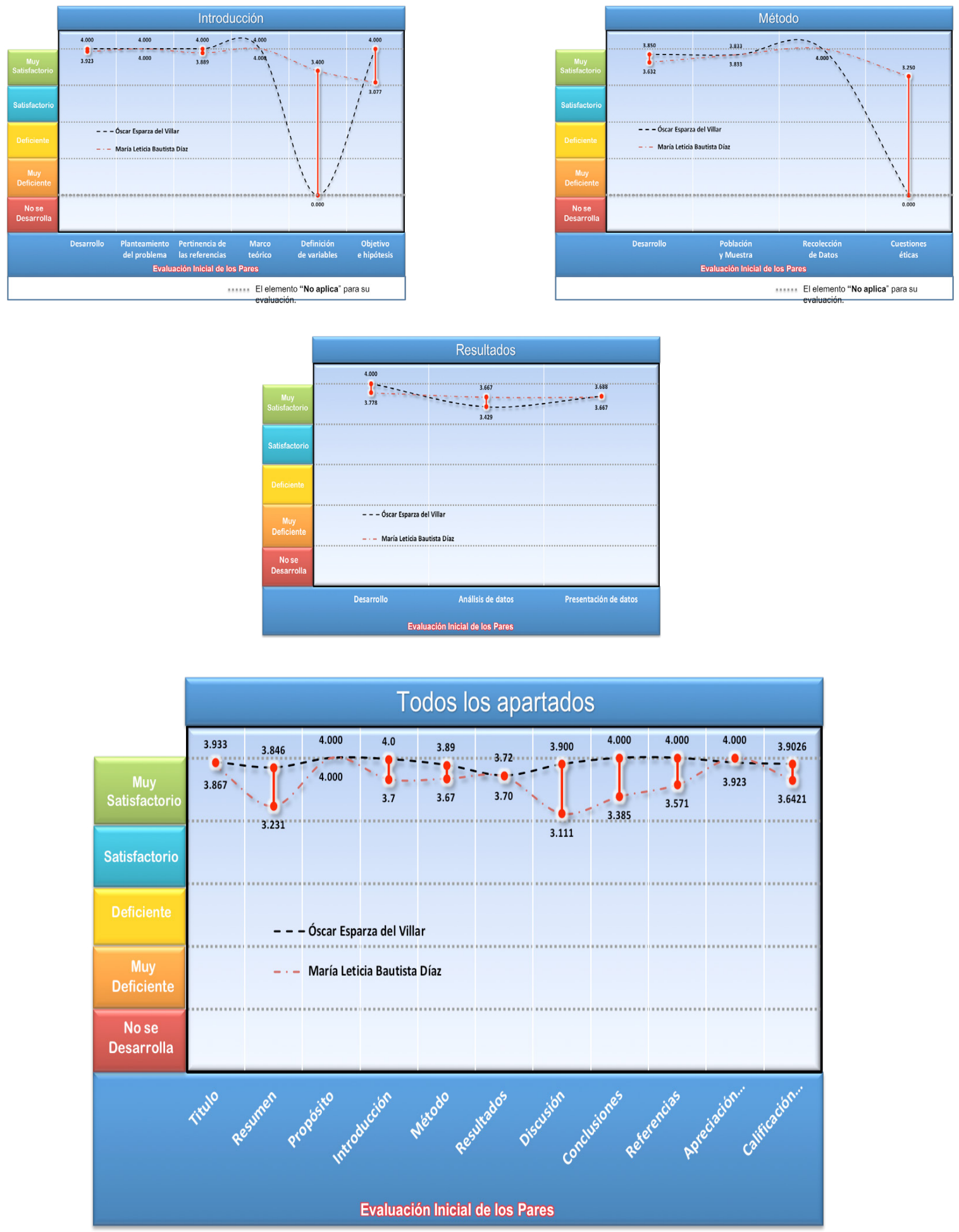


\section{Índice de Concordancia}

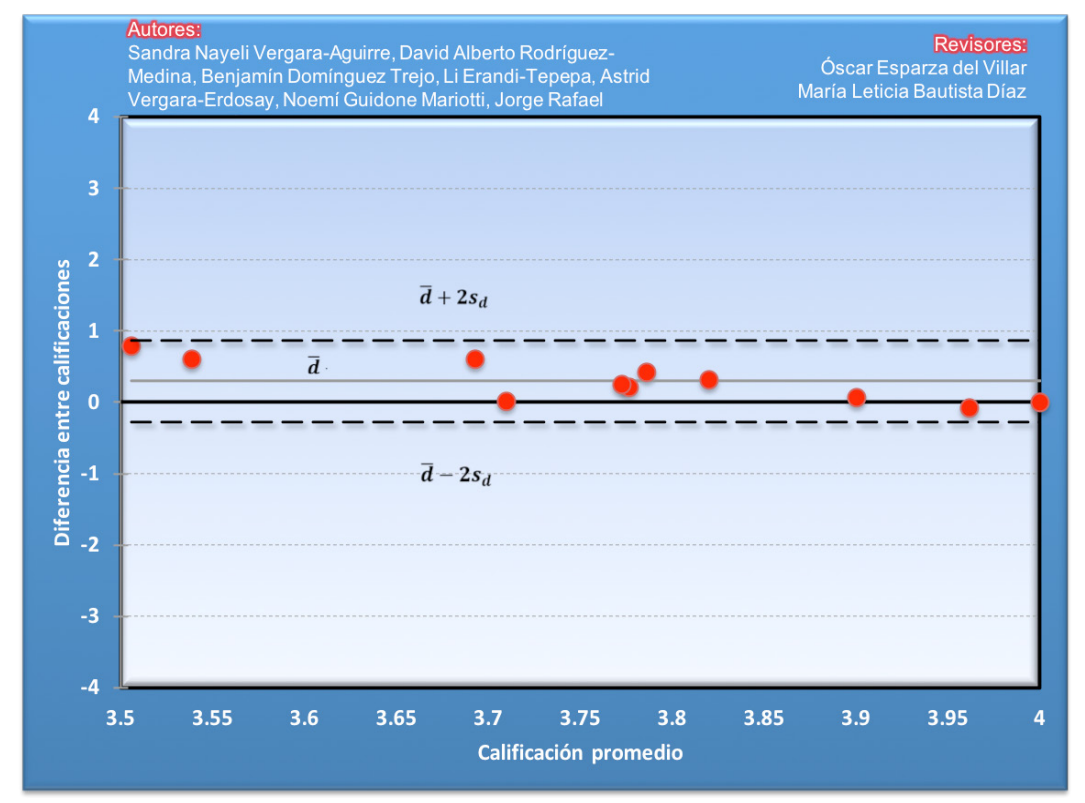

Índice de Acuerdo

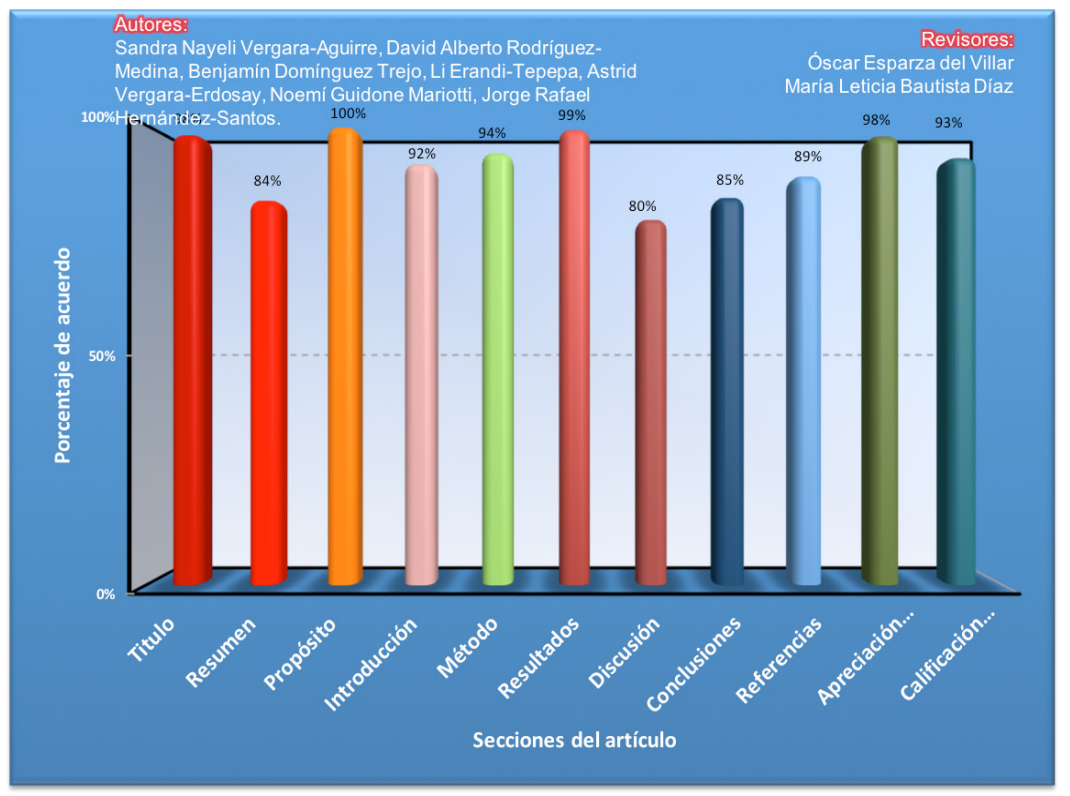




\section{Dimensión Cualitativa}

\begin{tabular}{|c|c|}
\hline Revisor 1 & Revisor 2 \\
\hline Óscar Esparza Del Villar & María Leticia Bautista Díaz \\
\hline \multicolumn{2}{|c|}{ Título/Autoría } \\
\hline Incluir el correo de los autores & Sin observaciones \\
\hline \multicolumn{2}{|c|}{ Resumen } \\
\hline Indicar con claridad el método y diseño del estudio & $\begin{array}{l}\text { En el apartado de conclusiones se sugiere referir qué im- } \\
\text { plicaciones psicológicas tienen estos hallazgos para los pa- } \\
\text { cientes con dolor crónico }\end{array}$ \\
\hline \multicolumn{2}{|c|}{ Próposito del Estudio } \\
\hline Lo considero satisfactorio. & $\begin{array}{l}\text { Se sugiere referir que la intervención psicológica la cons- } \\
\text { tituye la estimulación térmica (ya que también es suges- } \\
\text { tiva) y la analgesia hipnótica }\end{array}$ \\
\hline \multicolumn{2}{|c|}{ Introducción } \\
\hline $\begin{array}{l}\text { Establecer la pregunta de investigación de manera clara. } \\
\text { El marco teórico puede incluir los conceptos que am- } \\
\text { plian la información sobre los resultados encontrados. } \\
\text { Ejemplo del cyberbulling no se redacta con claridad. }\end{array}$ & $\begin{array}{l}\text { Se sugiere atender unas citas textuales y longitud de los } \\
\text { párrafos }\end{array}$ \\
\hline \multicolumn{2}{|c|}{ Método } \\
\hline Explicar el procedimiento del muestreo. & $\begin{array}{l}\text { En el caso del promedio de edad de los participantes, falta } \\
\text { la desviación estándar }\end{array}$ \\
\hline
\end{tabular}


Artículo Teórico | Estimulación térmica y analgesia fipnótica... Vergara-Aguirre, Rodríguez-Medina, et al

\begin{tabular}{|l|l|}
\hline \multicolumn{1}{|c|}{ Revisor 1 } & \multicolumn{1}{|c|}{ Revisor 2 } \\
\hline $\begin{array}{l}\text { Desultados } \\
\text { zación de las variables. }\end{array}$ & Que no inicien el aparado con hallazgos no significativos \\
\hline \multicolumn{2}{|c|}{ Discusión } \\
\hline $\begin{array}{l}\text { Interpretar los resultados del estudio a la luz de investi- } \\
\text { gaciones antecedentes. Abordar hipótesis alternativas. }\end{array}$ & $\begin{array}{l}\text { Se debe tomar en cuenta que los pacientes tenían dife- } \\
\text { rentes diagnósticos y se desconoce el tiempo del dolor }\end{array}$ \\
\hline \multicolumn{2}{|c|}{ Conclusiones } \\
\hline Redacción satisfactoria de la conclusión. & $\begin{array}{l}\text { Es necesario conocer cuáles son las implicaciones psico- } \\
\text { lógicas del estudio para la comunidad científica y para las } \\
\text { personas con DC }\end{array}$ \\
\hline \multicolumn{2}{|c|}{ Referencias } \\
\hline
\end{tabular}




\section{Historia del Proceso}

\section{EDITORIAL}

